\title{
Mysterious Mesenteric Mass- an Unusual Presentation of Hodgkin's Disease
}

\author{
Authors \\ Indu Manicketh ${ }^{1}$, Anuradha Ananthamurthy ${ }^{2}$, Namita Sinha ${ }^{3}$ \\ ${ }^{1} \mathrm{MD}$, Assistant Professor in Pathology, Vydehi Institute of Medical Sciences and Research Centre, EPIP \\ area, Whitefield, Bangalore, India \\ ${ }^{2}$ MD, Associate Professor in Pathology, St John's National Academy of Health Sciences, Sarjapur Road, \\ Bangalore, India \\ ${ }^{3} \mathrm{MD}$, Consultant radiologist, Columbia Asia Hospital, White field, Bangalore, India \\ Corresponding Author
}

\section{Dr Indu M}

No: 785, JJ hospital, Anna Nagar, Madurai-625020, Tamil Nadu, India

Email-drindu_m@yahoo.in, Phone number-09739138336

\begin{abstract}
Hodgkin's disease of the mesenteric lymphnode presenting as a mesenteric mass is an unusual clinical presentation. Due to the rarity of this clinical presentation, the diagnosis is seldom thought of. Radiological imaging studies play an important role in arriving at the right diagnosis. Mesenteric lymphomas can have a characteristic radiological appearance on CT imaging which is known as the 'Sandwich sign'. Herein we present a case of mesenteric Hodgkin's disease which mimicked a gastrointestinal stromal tumour. An accurate pre operative diagnosis helps in avoiding unnecessary surgical interventions and related morbidity.
\end{abstract} Keywords: Hodgkin's lymphoma, mesentery, RS cell, Sandwich sign

\section{Introduction}

Hodgkin's lymphoma presenting as a symptomatic mesenteric mass is an exceedingly rare presentation. A perusal of literature reveal that the commonest mesenteric lymphomas are non Hodgkin's lymphoma. We present an unusual case of Hodgkin's lymphoma involving multiple coalescent mesenteric lymph nodes masquerading as a gastrointestinal tumour both clinically and radiologically. Post operative histopathology and immunohistochemistry confirmed the diagnosis of Hodgkin's lymphoma. A retrospective review of the CT images revealed the "Sandwich sign' which is a characteristic radiological sign seen in mesenteric lymphomas.

\section{Case History}

A 76 year old lady presented to the surgical OPD with the complaints of intermittent dull aching abdominal pain for a duration of one month. The pain was localised to the right lower quadrant of the abdomen. She also had intermittent low grade fever, generalised fatigue, loss of weight and loss of appetite. She was hypothyroid, diabetic and hypertensive and was on medication for the same.

On general physical examination, the only significant clinical finding was pallor. Systemic examination was within normal limits. 


\section{Investigations}

The haematological work up showed Hemoglobin of $8.3 \mathrm{~g} / \mathrm{dl}$, total WBC count of 22980/cu mm. The differential count revealed neutrophilia of 92\%. The ESR was 110. Peripheral smear showed a normocytic hypochromic blood picture.

Liver function test was normal. Serum LDH was $132 \mathrm{U} / \mathrm{L}$

The chest X ray was normal. Upper GI endoscopy and colonoscopy were normal. Ultrasound of the abdomen showed a cystic lesion which was thought to be of ovarian origin.

CT scan abdomen and pelvis revealed a solid cystic lesion in the mesentery with thick shaggy walls with air fluid levels within the lesion and surrounding lymphadenopathy. A suspicious communication with the small bowel loop was noted. The radiological impression was that of a cavitating gastrointestinal stromal tumour (GIST) with ulceration. Bilateral ovaries and uterus appeared normal.

The patient was posted for a diagnostic laparotomy after the requisite pre operative investigations.

Intra operatively a mass was seen adherent to the loops of the ileum with bleeding from the tumour surface. Resection of the mass with the adjacent small bowel was performed followed by an ileo ileal anastamosis and peritoneal wash. The resected mass with the segment of intestine was sent for histopathological examination.

Gross examination showed a segment of small intestine with a mass on the serosal aspect measuring $8.5 \times 4.5 \times 3 \mathrm{~cm}$. The cut surface of the mass was lobulated, grey white and with areas of necrosis and hemorrhage (Figure 1). The attached appendix was unremarkable.

Microscopy revealed a polymorphous infiltrate of cells with classical Reed Sternberg (RS) cells as well as mononuclear, multi nucleate and mummified cells which were characterized by enlarged vesicular nuclei and prominent eosinophilic nucleoli in a background showing lymphocytes, eosinophils and plasma cells (Figure 2). Residual lymphoid tissue was identified at foci. Immunohistochemistry (IHC) showed the neoplastic RS cells staining positive with antibodies against vimentin, CD30, Pax 5 and Bcl2 and exhibited negative staining with anti CD 45, anti CD 20, anti CD 15 , EMA, ALK , CK and anti CD 68 antibodies (Figure 2 inset). The IHC was performed using the polymer technique.

The histomorphology and immunohistochemical profile was consistent with the diagnosis of a classical Hodgkin's disease of mixed cellularity type.

Staging was done and the patient was found to have Stage IB disease. She was started on ABVD (Adriamycin, Bleomycin, Vinblastine, Dacarbazine) regimen. On follow up at the end of 4 months the patient had finished 3 cycles of chemotherapy and was free of disease.

A retrospective review of the radiological images revealed the so called "Sandwich sign" characteristic of mesenteric lymphomas (Fig 3).

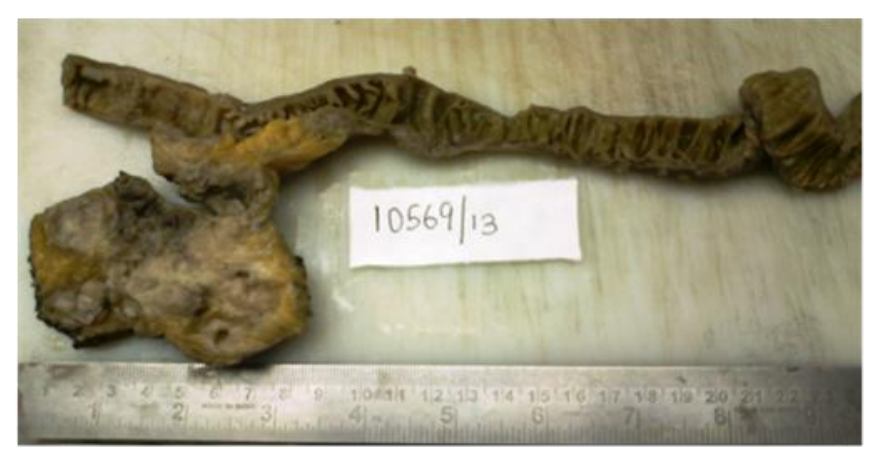

Figure 1: Lobulated mass arising from the serosal aspect of the small intestine. 


\section{JMSCR Vol||3||Issue||9||Page 7513-7516||September}

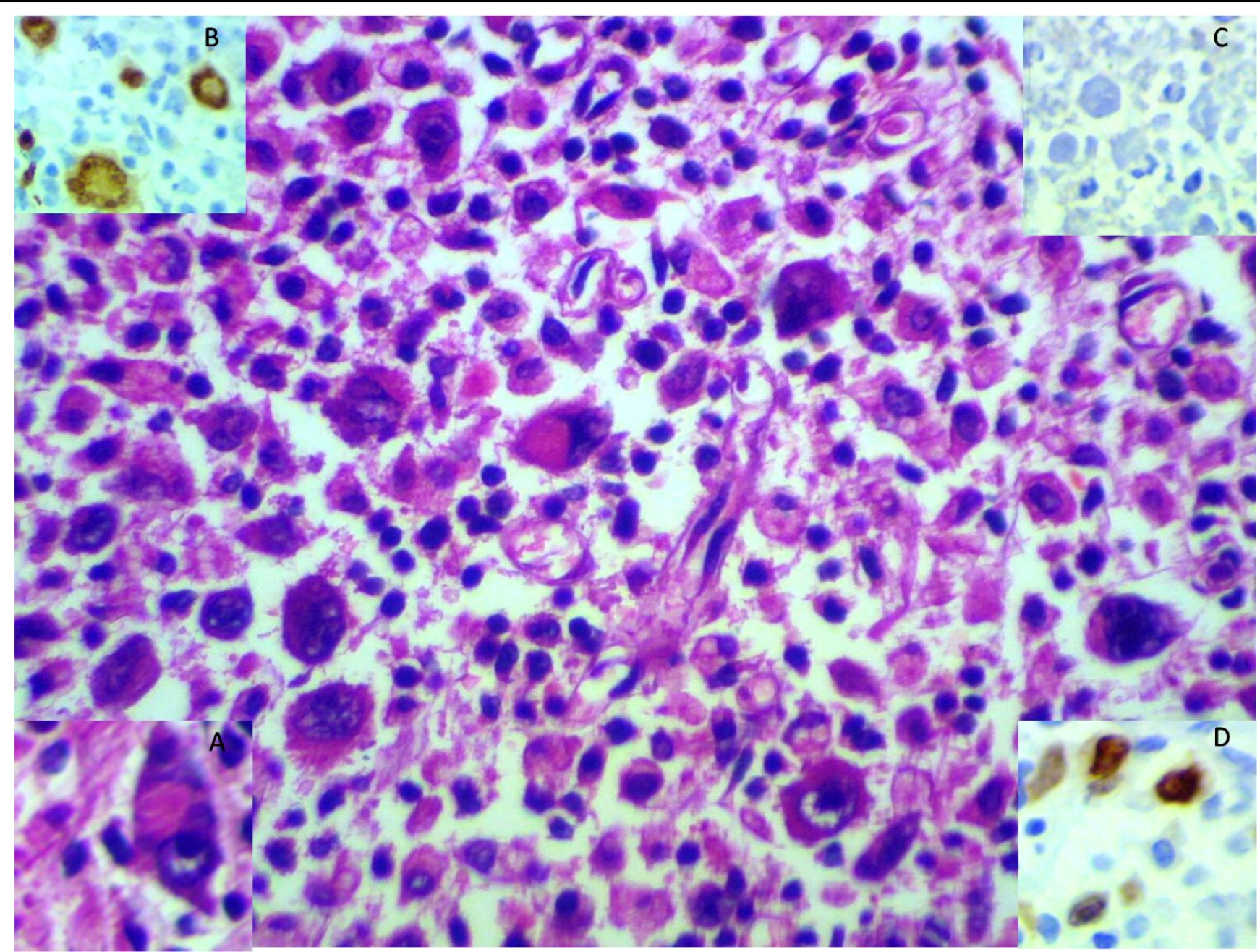

Figure 2: Mononuclear RS cells in a background showing eosinophils, neutrophils and plasma cells. Inset A- Classical RS cell, Inset B,C,D- RS cells positive for CD 30 , negative for LCA and positive for Pax -5 .

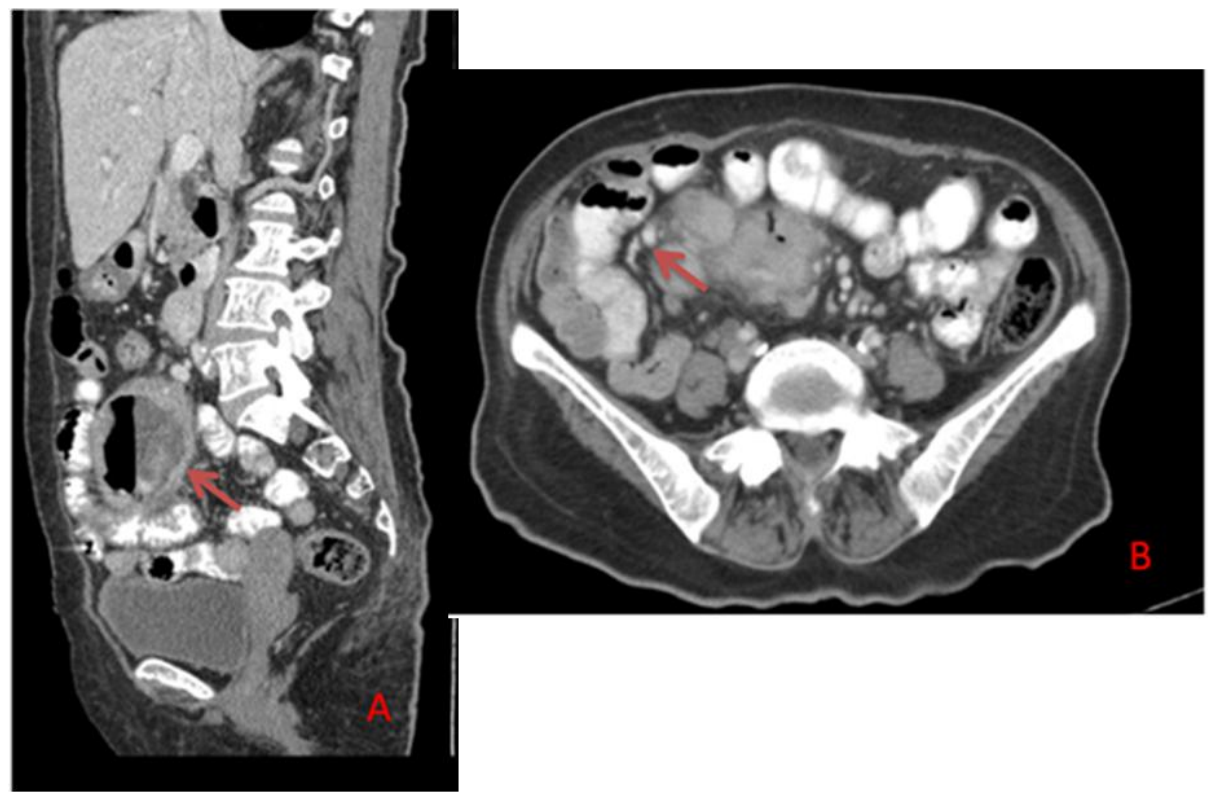

Figure 3: A- Reconstructed sagittal post contrast CT images showing a cavitating mesenteric lesion with air fluid levels situated in close proximity to the bowel (arrow) B- Axial post contrast CT image showing a lesion being traversed by a branch of superior mesenteric artery, the so called Sandwich sign (arrow). 


\section{Discussion}

Primary extra nodal and sub diaphragmatic disease is rare in Hodgkin's disease. ${ }^{1}$ Hodgkin's disease presenting primarily as a mesenteric mass in the absence of any other nodal involvement is an unusual clinical presentation. The mesenteric mass in this case was a mesenteric lymph nodal mass which was adherent to the small bowel and infiltrating it. A perusal of the English literature using the search terms of Hodgkin's lymphoma and mesenteric lymph nodes yielded only a handful of case reports. One such case was that of a 45 year old man who presented with acute abdomen and found to have Hodgkin's disease involving the ascending colon and mesenteric lymph nodes. ${ }^{2}$ Concurrent occurrence of mesenteric Hodgkin's lymphoma and colonic adenocarcinoma has been reported. ${ }^{3}$ In these cases Hodgkin's disease was detected incidentally in the lymphnodes in specimens resected for carcinoma of the colon.

In our case, the neoplasm could possibly represent a lymph nodal mass formed by a conglomerate of mesenteric lymph nodes and the presence of residual lymphoid tissue within the neoplasm confirms this fact. However the lymphoid nature of the mass was difficult to discern macroscopically. The large size, areas of necrosis and the close proximity to the serosal aspect of the bowel with no luminal communication mimicked a gastrointestinal stromal tumour/ mesenchymal tumour.

'Sandwich sign' in ultrasonography and computed tomography has been described in mesenteric lymphomas which was present in our case also. However this finding was identified only on the review of the radiological films. The presence of a lobulated confluent mass encasing the superior mesenteric artery and veins producing a sandwichlike appearance is called as the Sandwich sign. ${ }^{4}$ This sign is characteristic though not specific for mesenteric lymphomas. ${ }^{5}$ Mueller et al in their short series of seven cases have described this sign and have emphasised the importance of the correct pre operative recognition of this sign which could avoid unnecessary therapeutic interventions. The pre operative recognition of this radiological sign would have proved beneficial in our case as the patient was an elderly female with co morbidities and anaesthetic risk factors.

\section{Acknowledgement}

The authors thank the technical staff of St John's medical college hospital for the histopathological and IHC sections.

\section{References}

1. Miller TP, Byrne GE, Jones SE. Mistaken clinical and pathologic diagnoses of Hodgkin's disease: a Southwest Oncology Group study. Cancer Treat Rep 1982;66:645-651.

2. Gupta D, Sharma A, Dwary A, Kark AK. Hodgkin lymphoma involving ascending colon and mesenteric lymphnodes: A rare entity. Indian J Cancer. 2009 AprJun;46(2):175-6.

3. Petros JG, Argy O. Hodgkin's disease in the mesenteric lymph nodes in a patient with colon carcinoma. Mt Sinai J Med. 1990 Nov;57(6):368-70.

4. Mueller PR, Ferrucci JT Jr, Harbin WP, Kirkpatrick RH, Simeone JF, Wittenberg J. Appearance of lymphomatous involvement of the mesentery by ultrasonography and body computed tomography: the "sandwich sign". Radiology. 1980 Feb;134(2):467-73.

5. Lien WC, Huang SP, Liu KL, Chang JH, Lai TI, Liu YP et al. The sandwich sign of non lymphomatous origin. J Clin Ultrsound. 2009 May;37(4):212-4 